\title{
LAB: Lightweight Adaptive Broadcast Control in DSRC Vehicular Networks
}

\author{
Linsheng Ye, ${ }^{1}$ Linghe Kong ${ }^{D},{ }^{1}$ Kayhan Zrar Ghafoor, ${ }^{1}$ \\ Guihai Chen, ${ }^{1}$ and Shahid Mumtaz ${ }^{(\mathbb{D}}{ }^{2}$ \\ ${ }^{1}$ Shanghai Jiao Tong University, China \\ ${ }^{2}$ Instituto de Telecomunicacoes, Portugal \\ Correspondence should be addressed to Linghe Kong; linghe.kong@sjtu.edu.cn
}

Received 26 April 2018; Revised 16 July 2018; Accepted 5 August 2018; Published 13 August 2018

Academic Editor: Dongyao Jia

Copyright (C) 2018 Linsheng Ye et al. This is an open access article distributed under the Creative Commons Attribution License, which permits unrestricted use, distribution, and reproduction in any medium, provided the original work is properly cited.

\begin{abstract}
The Industrial Internet of Things (IIoT) is the use of Internet of Things (IoT) technologies in manufacturing. The vehicular ad hoc networks (VANETs) are a typical application of IIoT. Benefiting from Dedicated Short-Range Communication (DSRC) technology, vehicles can communicate with each other through wireless manner. Therefore, road safety is able to be greatly improved by the broadcast of safety messages, which contain vehicle's real-time speed, position, direction, etc. In existing DSRC, safety messages are broadcasted at a fixed frequency by default. However, traffic conditions are dynamic. In this way, there are too many transmission collisions when vehicles are too dense and the wireless channel is underused when vehicles are too sparse. In this paper, we address broadcast congestion issue in DSRC and propose lightweight adaptive broadcast (LAB) control for DSRC safety message. The objectives of LAB are to make full use of DSRC channel and avoid congestion. LAB meets two key challenges. First, it is hard to adopt a centralized method to control the communication parameters of distributed vehicles. Furthermore, the vehicle cannot easily acquire the channel conditions of other vehicles. To overcome these challenges, channel condition is attached with safety messages in $\mathrm{LAB}$ and broadcast frequency is adapted according to neighboring vehicles' channel conditions. To evaluate the performance of $\mathrm{LAB}$, we conduct extensive simulations on different roads and different vehicle densities. Performance results demonstrate that LAB effectively adjusts the broadcast frequency and controls the congestion.
\end{abstract}

\section{Introduction}

Internet of Things (IoT) is an ecosystem of connected physical objects such as home appliances, vehicles, and robots, so that they have ability to access the Internet and communicate with each other [1]. Industrial Internet of Things (IIoT) is an application of the IoT to the manufacturing industry, which is believed to promote the development of manufacturing industry [2]. IIoT is also the foundation of industry 4.0 $[3,4]$. Plenty of modern technologies are integrated into IIoT, such as cloud computing [5], wireless networks [6], artificial intelligence [7], autonomous vehicles [8], and data analysis, which makes it more powerful. The vehicular ad hoc networks (VANETs) [9] are a typical application of IIoT and make vehicles have the ability to communicate with each other. In recent years, Dedicated Short-Range Communication (DSRC) [10] is an emerging technology in VANETs, which provides the communication capability among vehicles [11]. It is reported that connected vehicles can help to avoid $74 \%$ of car crashes, which would save tens of thousands of lives and billions of dollars every year [12]. In addition, the US Federal Communication Commission (FCC) allocated $75 \mathrm{MHz}$ licensed spectrum at $5.9 \mathrm{GHz}$ for DSRC, which can be used exclusively for vehicle-to-vehicle $(\mathrm{V} 2 \mathrm{~V})$ and vehicle-to-infrastructure (V2I) communications [13]. The DSRC spectrum is divided into 7 channels and the control channel $(\mathrm{CCH})$ is exclusively reserved for safety communications [14].

Periodical broadcast of vehicles' safety messages is a key factor of road safety improvement. To let all neighboring vehicles within the transmission range know the condition of a vehicle, this vehicle broadcasts its safety messages to all single-hop vehicles on control channel. These safety messages provide detailed information like speed, position, direction, 
acceleration, brake status, etc. [15]. The safety messages are broadcasted periodically, usually at a fixed frequency. The default periodicity is 0.1 seconds [16], as the recommendation of industry.

DSRC uses carrier sense multiple access with collision avoidance (CSMA/CA) at MAC layer as the fundamental multiaccess scheme [17]. When the density of vehicles within an area is too high, the channel is full of safety messages and collision rate increases rapidly. In this case, the vehicles cannot decode received collided safety messages and it becomes hard for the vehicle to know the status of neighbors. On the other hand, when the density of vehicles is relatively low, the channel is underused. To make full use of the channel resource, it is better if vehicles broadcast more safety messages. Therefore, broadcasting at fixed frequency does not fit in dynamic traffic circumstance. This issue becomes the motivation of our work.

To tackle this issue, there exist two challenges caused by the DSRC broadcast mechanism. First, vehicles are distributed to and independent of each other. So it is hard to use a centralized method to control the broadcast periodicity of all vehicles. Furthermore, since a vehicle does not know the channel conditions of other vehicles, it is not easy to set an accurate periodicity with only individual information.

In this paper, we propose a novel method, named lightweight adaptive broadcast (LAB) control, for DSRC safety message to make full use of channel and avoid channel congestion. LAB uses channel detector to assess channel condition and this information will be attached along with the safety message. Broadcast manager analyses neighbors' channel conditions and adjusts broadcast frequency (broadcast frequency represents the transmission frequency of safety message in this paper) correspondingly. In this way, the broadcast frequency is reduced when vehicle density is high and the broadcast frequency is increased when vehicle density is low. Furthermore, the challenges are overcome in a distributed way, because vehicles can acquire neighbors' channel conditions and make the decision of periodicity by themselves.

The contributions of this work are as follows:

(i) We propose and study a problem in DSRC that the broadcast frequency of safety messages should be carefully adjusted in dynamic traffic circumstance.

(ii) We propose a novel lightweight adaptive broadcast control (LAB) framework for DSRC safety message. LAB is a completely distributed scheme and vehicles broadcast their safety messages adaptively and efficiently under any conditions.

(iii) We conduct extensive simulations to evaluate LAB. Performance results demonstrate that $\mathrm{LAB}$ is an effective broadcast scheme. It works well on different vehicle densities and average received safety messages are up to 3 times compared with standard DSRC method. Moreover, it converges to global optimal state within 10s.

The rest of paper is organized as follows. In Section 2, we review the related work. Section 3 is problem statement of this paper. Section 4 presents the design details of our proposed scheme LAB. We evaluate performance of LAB in Section 5. Section 6 concludes our paper.

\section{Related Works}

In literature, plenty of solutions are proposed to control congestion in DSRC, which aim at enhancing the performance of vehicular networks.

Fei Ye et al. [18] proposed the congestion control and power control strategy that maximizes the efficiency, after analysing the broadcast efficiency under Rayleigh fading channel.

Soufiene Djahel et al. [19] proposed an algorithm that consists of three phases: assign priority to the safety messages; detect congestion; adjust transmit power and safety message transmission rate.

Lv Humeng et al. [20] proposed a distributed safety message frequency control algorithm adjusting the broadcast frequency according to the current network condition.

Mohamed Salah et al. [21] presented a congestion control algorithm based on the concept of dynamic priorities-based scheduling, to provide a reliable and safe communications system for VANETs.

M. A. Benatia et al. [22] proposed a Markov chain model to control congestion, which consists of four steps: priority assignment, buffer monitoring, congestion detection phase, and beacon transmission rate adjustment.

Bilal Munir Mughal et al. [23] proposed a conceptual view of a congestion control scheme which adjusts transmission rate and transmission power simultaneously for optimal congestion control.

Gaurav Bansal et al. [24] proposed a method which is based on LIMERIC [13] algorithm and achieves weighted fair message rate congestion control.

Tessa Tielert et al. [25] presented a design methodology for congestion control. Furthermore, a resulting rate adaption oriented protocol named PULSAR is proposed with the description and evaluation.

Gaurav Bansal et al. [26] proposed a congestion control method called EMBARC which adjusts the safety message transmission rate based on both channel load and vehicular dynamics.

In this work, we focus on a solution which can not only control channel congestion, but also make full use of channel resource and ensure fairness.

\section{Problem Statement}

In order to ensure road safety, vehicles equipped with DSRC devices broadcast their safety messages carrying their safety status among neighboring vehicles. By default, broadcast frequency is a fixed value, which cannot make full use of the channel and may lead to congestion. So it is necessary to propose a tailored adaptive broadcast control for DSRC safety message.

3.1. Notations. We summarize the notations of this paper as follows. We use $R$ to denote a list of channel busy rates, and 
TABLE 1: Summary of notations.

\begin{tabular}{lc}
\hline Notation & Definition \\
\hline$R$ & a list of vehicles' channel busy rates \\
$r_{i}$ & channel busy rate of vehicle $i$ \\
$r$ & channel busy rate \\
$r^{*}$ & optimal channel busy rate \\
$v_{i}$ & vehicle $i$ \\
$s_{i}$ & safety message $i$ \\
$f$ & broadcast frequency \\
$f^{\prime}$ & adjusted broadcast frequency \\
$F_{\text {low }}$ & frequency lower bound \\
$F_{\text {high }}$ & frequency upper bound \\
twnd & time window \\
$T_{\text {idle }}$ & idle time in a twnd \\
$\alpha$ & step size \\
\hline
\end{tabular}

$r_{i}$ is the item of $R$. $f$ denotes the broadcast frequency. twnd denotes the time window. Other key notations in this paper are listed in Table 1.

3.2. Problem Formulation. Our objectives are to make full use of channel, avoid channel congestion, and guarantee fairness. To formulate these three objectives, we define $r$ as the ratio of channel busy state to express the channel condition; use $T_{i d l e}$ to denote idle time of channel in a time window twnd; use $r^{*}$ to denote optimal channel busy rate. To make full use of channel, $T_{\text {idle }}$ should be minimized; to avoid channel congestion, it should be guaranteed that the average of neighboring vehicles' channel busy rates approaches $r^{*}$; to guarantee fairness, the standard deviation of $r$ should be minimized, so that each vehicle can have same chance to broadcast its safety messages. Therefore, the objectives can be formulated as

$$
\begin{gathered}
\min T_{i d l e} \\
\min \sigma(r) \\
\bar{r}=r^{*}
\end{gathered}
$$

To make it adaptive, broadcast frequency should be adjusted according to real-time channel condition. Furthermore, to prevent the broadcast frequency from getting too high or too low, we should limit it within a range. It will not get more benefits from too high broadcast frequency while it is energy wasted. Meanwhile, for basic requirement of safety message broadcasting, broadcast frequency should have a minimum limit. In this way, we use $F_{\text {high }}$ to denote frequency upper bound and $F_{\text {low }}$ to denote lower bound. Based on the above analysis, we formulate the problem as

$$
\begin{gathered}
\min T_{\text {idle }} \\
\min \sigma(r) \\
\bar{r}=r^{*}
\end{gathered}
$$

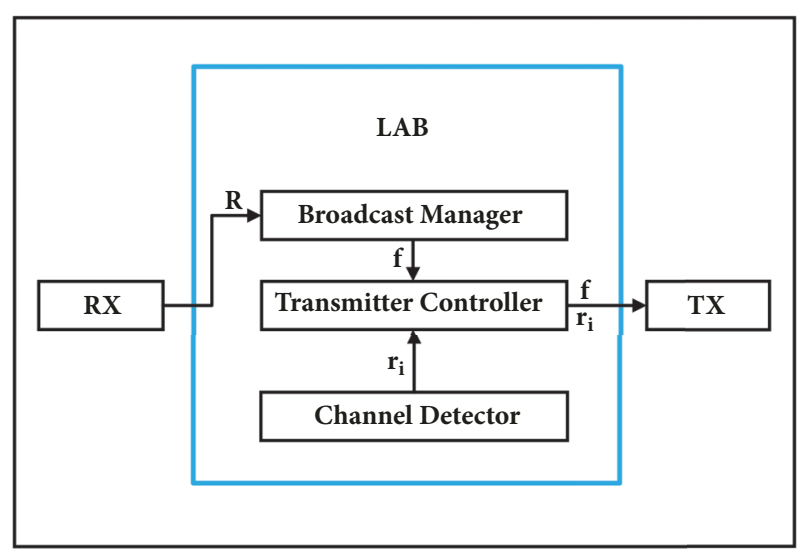

FIgURE 1: Overview of LAB.

$$
\begin{gathered}
F_{\text {low }}<f<F_{\text {high }} \\
0 \leq r_{i} \leq 1
\end{gathered}
$$

\section{Design of LAB}

In this section, we introduce the design of our proposed lightweight adaptive broadcast (LAB) control in detail. Firstly, we present the overview on LAB. Then, we describe the channel detection mechanism. At last, we introduce the details of broadcast frequency control strategy.

4.1. Design Overview. To solve the problem formulated in (2), we design LAB, whose overview is shown in Figure 1. LAB has three core modules: broadcast manager, transmitter controller, and channel detector.

Channel detector is able to detect channel state and channel condition is conveyed to transmitter by transmitter controller. When transmitter gets channel condition, it will attach channel condition to safety messages, which contain safety information and other information. The format of the $\mathrm{LAB}$ frame is shown in Figure 2.

Broadcast manager will get the messages from receiver and fetch the carried contents. Then broadcast manager will analyse the channel conditions of nearby vehicles according to received messages, because channel condition is attached in these messages. At last, broadcast manager will make an adjustment for broadcast frequency and send the adjustment request to transmitter controller, to change broadcast frequency.

Transmitter controller controls the behavior of transmitter and transfers channel condition to transmitter.

Because the decision made by broadcast manager is based on channel condition, the objectives of making full use of channel and avoiding channel congestion are satisfied. At the same time, the objective of fairness is also satisfied for the consideration of nearby vehicles' channel conditions. The deeper analysis can be found in Section 4.3.

In the following subsections, we introduce the details of our design. 


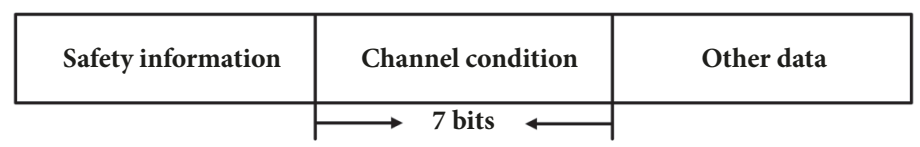

FIGURE 2: Packet format of safety message.

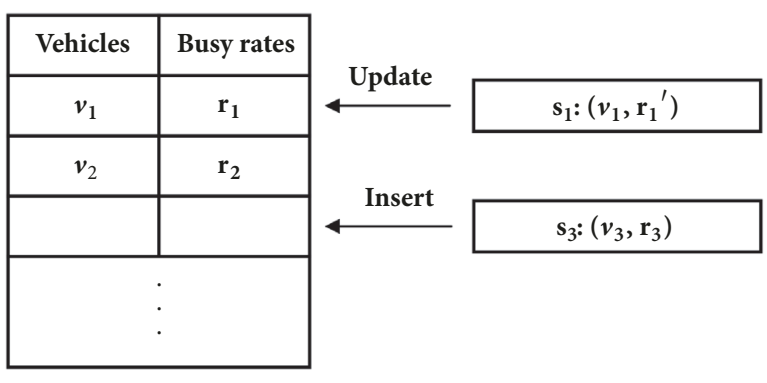

FIgure 3: Busy rate table.

4.2. Channel Detection. Channel condition can be quantified by many indicators. Channel detector chooses the channel busy rate, which is the ratio of channel when it is in a busy state. Busy state means channel is not idle and it may be transmitting, receiving, etc. Channel detector periodically detects channel state to quantify channel busy rate.

To quantify the busy state of the channel, a typical way is to conduct multiple sampling in a time slot. Because the busy state of the channel is dynamic, it is important to choose a suitable time slot. Channel detector detects the channel state every $10 \mathrm{~ms}$ and maintains a time window $(t w n d)$. The length of time window is set to 1s by default. Every time it finds channel in a busy state within twnd, channel detector will record it. At the end of twnd, channel detector will send the channel busy rate to transmitter and start a new twnd.

It is worth noting that only 7 bits are appended to safety message, for conveying busy rate, as shown in Figure 2. The reason is that channel detector has 100 sampling points for each twnd and there are only 100 possible values for busy rate. Therefore, it contributes to LAB's lightweight features because it almost does not increase the communication overhead.

4.3. Broadcast Frequency Control. LAB adjusts broadcast frequency of transmitter according to neighboring vehicles' channel conditions. Whenever the receiver of vehicle receives a safety message from a neighbor, the content carried by this message will be fetched by broadcast manager.

Broadcast manager maintains a busy rate table and a time window $(t w n d)$. Every time a vehicle receives a safety message, broadcast manager will record channel busy rate into busy rate table, which indicates the channel condition of the vehicle that sends this message. As shown in Figure 3, if a safety message carries channel busy rate of a new vehicle, broadcast manager will insert a new entry into busy rate table; or it will update corresponding entry. Moreover, this busy rate table only records channel busy rates in current twnd and it will be reset at the end of twnd. Reset mechanism

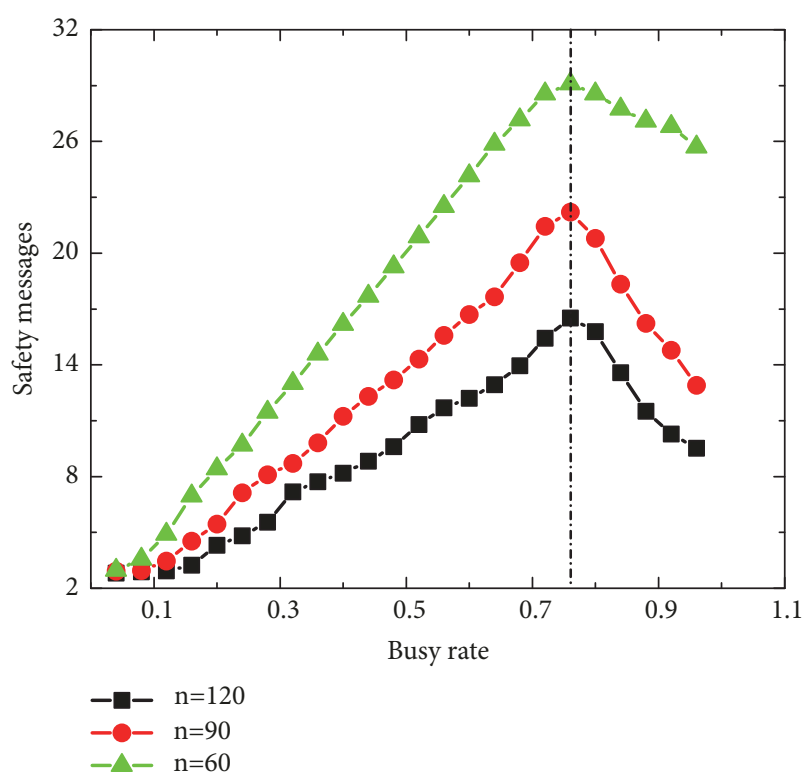

FIGURE 4: Safety messages on different channel busy rates.

ensures that busy rate table only maintains the vehicles within transmission range in each $t w n d$. The frequency adjustment decisions should be based on these vehicles. The overhead of reset is small because there are only hundreds of vehicles in transmission range at most typically.

One goal of LAB is to let vehicles receive valid safety messages as many as possible. We find that there exists an optimal busy rate $r^{*}$, in which the number of valid safety messages received by vehicle reaches the maximum value. It is easy to prove the existence of $r^{*}$. When the busy rate is close to zero, the channel is nearly idle and the vehicle receives few safety messages. On the contrary, when the busy rate is close to 1 , the channel is too busy and full of safety messages, which means that most of messages are collided and the vehicle receives few valid safety messages. From the previous analysis, we can conclude the existence of an optimal channel busy rate $r^{*}$. We simulate some communication scenarios to verify this conclusion, as shown in Figure 4. We place $n$ continuously moving vehicles at the crossroads in ns-3 [27]. $n$ changes in each scenario and vehicles broadcast their safety messages in different frequencies. We estimate the number of packets received by each vehicle and corresponding channel busy rate. Figure 4 shows the relation between channel busy rate and average safety messages received from neighbors. We find that when busy rate reaches to about 0.76 , vehicle gets most safety messages from neighbors in each scenario.

At the end of current twnd, broadcast manager will analyse maintained busy rate table and adjust broadcast frequency 
Input: Current broadcast frequency: $f$, Frequency lower bound: $F_{l o w}$, Frequency upper bound: $F_{\text {high }}$

Output: Adjusted broadcast frequency: $f^{\prime}$

(1) $f^{\prime}=f+\left\lceil\alpha\left(r^{*}-r\right)\right\rceil$;

(2) if $f^{\prime}<F_{L}$ then

(3) $f^{\prime}=F_{L}$;

(4) if $f^{\prime}>F_{H}$ then

(5) $\quad f^{\prime}=F_{H}$

(6) return $f^{\prime}$

Algorithm 1: LAB: broadcast frequency control.

correspondingly. Frequency control algorithm should meet the objectives and should not break the limitations shown in (2). Therefore, we propose a control algorithm shown as Algorithm 1.

LAB adopts gradient descent method to adjust broadcast frequency:

$$
f^{\prime}=f+\left\lceil\alpha\left(r^{*}-r\right)\right\rceil
$$

In the above equation, when the frequency converges to a value where the neighbors' average busy rate is equal to $r^{*}$, vehicle receives maximum safety messages.

It is worth mentioning that frequency should not break upper bound and lower bound. The reason for setting upper bound of broadcast frequency is that vehicles benefit less from broadcasting too many safety messages per second and it is energy wasted. Meanwhile the reason for setting lower bound of broadcast frequency is that vehicles must ensure a minimum broadcast frequency to spread their safety states.

In this way, vehicles can make full use of the channel to broadcast their safety messages. At the same time, the objective of fairness is guaranteed because LAB is a negative feedback process, whose causal chain is as follows:

$$
f_{i} \uparrow \Longrightarrow r_{\text {neighbors }} \uparrow \Longrightarrow f_{i} \downarrow
$$

Therefore, all vehicles have the same rights to broadcast their safety messages, which means the broadcast frequencies of single-hop neighbors are in a roughly same level.

On the contrary, a positive feedback will be trigged if control algorithm only uses vehicle's own channel busy rate to control frequency. The causal chain is shown as follows:

$$
f_{i} \uparrow \Longrightarrow r_{\text {neighbors }} \uparrow \Longrightarrow f_{\text {neighbors }} \downarrow \Longrightarrow r_{i} \downarrow \Longrightarrow f_{i} \uparrow
$$

Whenever broadcast manager decides to adjust broadcast frequency, it sends adjustment request to transmitter controller that controls the behavior of transmitter directly. Transmitter controller will adjust broadcast frequency of transmitter according to broadcast manager's decision. At last, transmitter will broadcast safety messages in specified frequency. Furthermore, these safety messages carry vehicle's safety information got from safety system of vehicle and channel condition got from channel detector.

\section{Evaluation}

To validate the performance of LAB, we use ns-3 [27] to simulate vehicle's broadcasting on different scenarios. We
TABLE 2: Simulation setting.

\begin{tabular}{lc}
\hline Parameters & Values \\
\hline Number of lanes & 3 \\
Lane width & $3.2 \mathrm{~m}$ \\
Transmission range & $300 \mathrm{~m}$ \\
Data rate & $3 \mathrm{Mbps}$ \\
Communication frequency & $5.9 \mathrm{GHz}$ \\
Packet length & $200 \mathrm{Bytes}$ \\
DSRC broadcast frequency & $10 \mathrm{~Hz}$ \\
$F_{\text {low }}$ & $5 \mathrm{~Hz}$ \\
$F_{\text {high }}$ & $30 \mathrm{~Hz}$ \\
$r^{*}$ & 0.76 \\
twnd & $1 \mathrm{~s}$ \\
$\alpha$ & 10 \\
\hline
\end{tabular}

generate vehicles' mobility traces using SUMO [28]. LAB is compared with standard DSRC method, unfair control method which adopts the vehicle's own channel busy rate to control frequency, and a distance based control method (denoted as DIST by us) proposed by Fallah et al. [29]. We create three types of roads in SUMO, which are crossroads, box road, and straight road. All roads in this simulation are bidirectional with three lanes in each direction. We use tworay ground propagation loss model in ns-3 setting. We run the simulations 100 s for each case. The data rate is set to $3 \mathrm{Mbps}$, which is the default data rate of broadcast in DSRC [30]. The settings of other parameters are summarized in Table 2. The following subsections are the details of evaluation results.

5.1. Performance on Different Densities. We test performance of LAB on different densities. Vehicles are moved in a crossroad being $1 \mathrm{~km}$ in each direction and vehicle number ranges from 20 to 380 .

Firstly, we test average safety messages received by each vehicle. The result is shown as Figure 5. We can easily find that LAB is better than standard DSRC and it receives more safety messages, because LAB controls broadcast frequency adaptively. Furthermore, the performance of LAB is similar to unfair control method. When vehicle number is larger than 220, DIST performs a bit better than LAB because DIST can reduce the transmission range. 


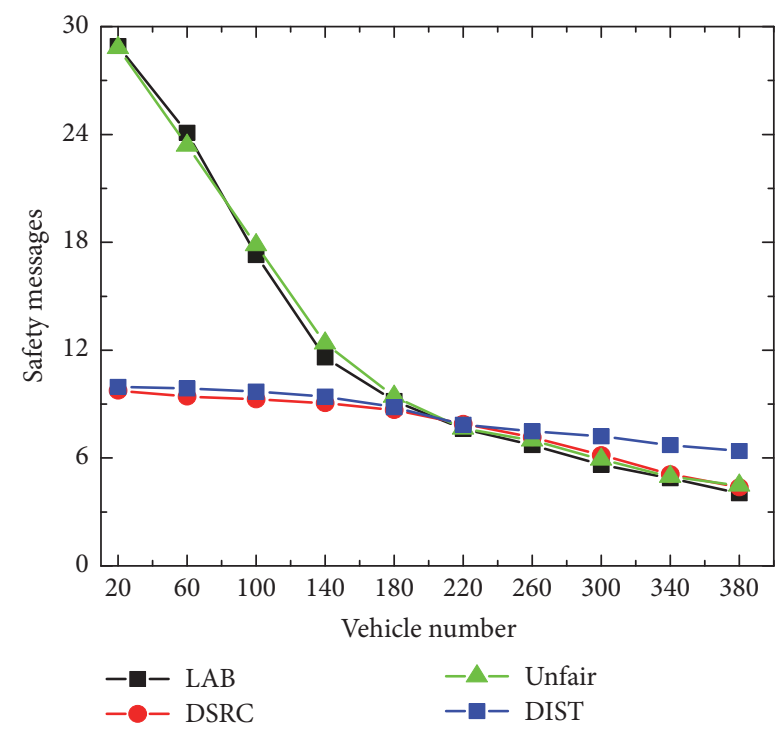

FIGURE 5: Safety messages on different vehicle densities.

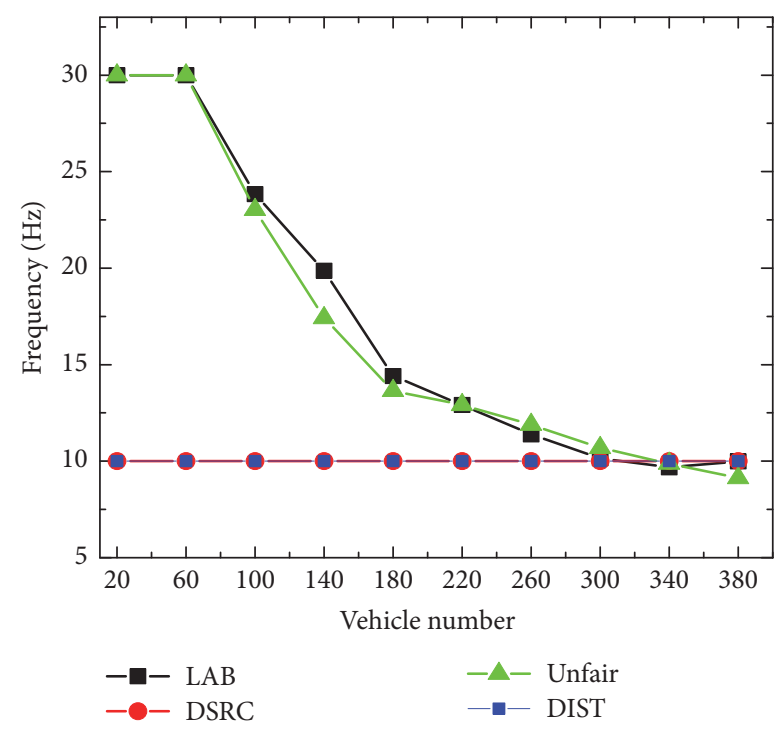

FIGURE 6: Broadcast frequency on different vehicle densities.

Figure 6 shows the average broadcast frequency on different vehicle densities. LAB's broadcast frequency drops from upper bound to lower bound with the increase of vehicle density while standard DSRC and DIST have no change. The curve of unfair method is also similar to LAB. The first two points of $\mathrm{LAB}$ are at frequency upper bound $30 \mathrm{~Hz}$ because the channel is not fully utilized at that time.

It is worth mentioning that although the global performance is roughly the same between LAB and unfair method, we can find the difference between them regarding fairness in next section.

5.2. Fairness. Then, we test whether each vehicle is treated fairly in LAB and it is compared with unfair control method.

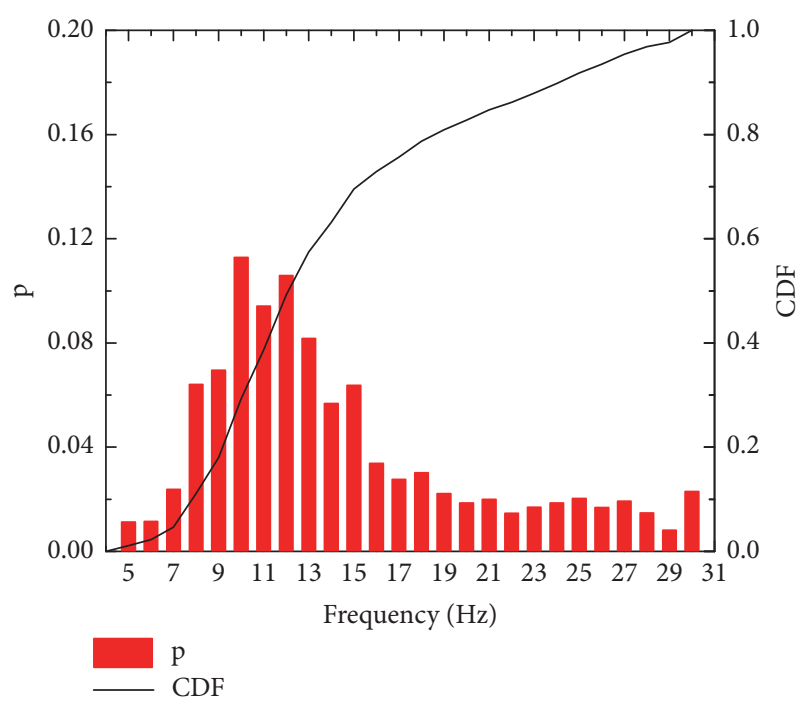

FIGURE 7: CDF of LAB method.

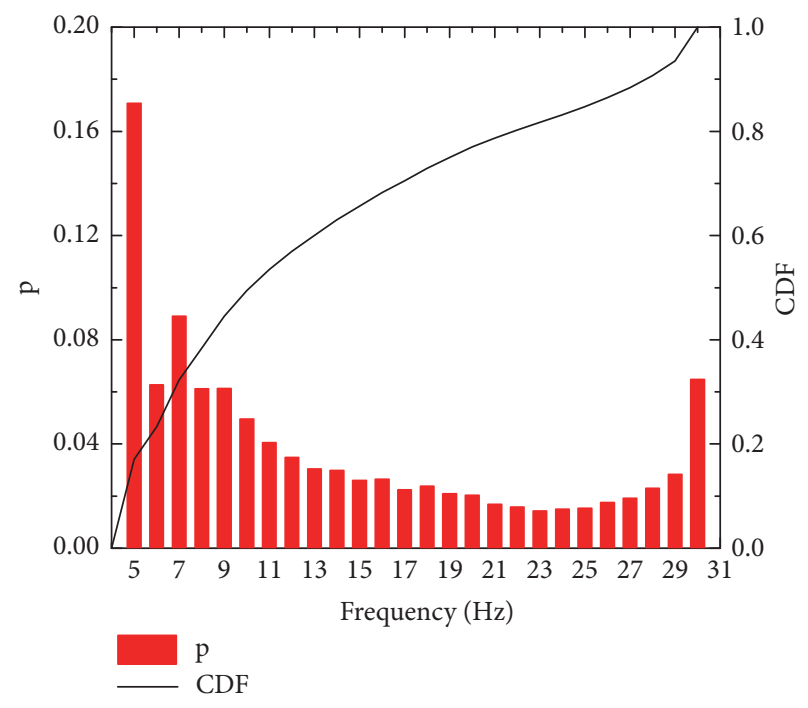

FIGURE 8: CDF of unfair method.

In this case, 180 vehicles are moved in a crossroad being $1 \mathrm{~km}$ in each direction.

Figure 7 shows LAB's cumulative distribution function (CDF) and probability distribution of each vehicle's broadcast frequency. Figure 8 is for unfair method.

As shown in Figure 7, frequency of each vehicle mainly appears at median of all vehicles' frequencies. However, in Figure 8, frequency mainly appears at $F_{\text {high }}$ and $F_{\text {low }}$. In this way, it can be concluded that LAB is more fair, which is the most important advantage compared with unfair method.

5.3. Time Trend. In this subsection, we check the performance changes of LAB over time, compared with standard DSRC method and unfair control method. In this test, 100 vehicles are moved in a crossroad being $1 \mathrm{~km}$ in each direction.

Firstly, we test changes of average received safety messages over time. The result is shown as Figure 9. We find that 


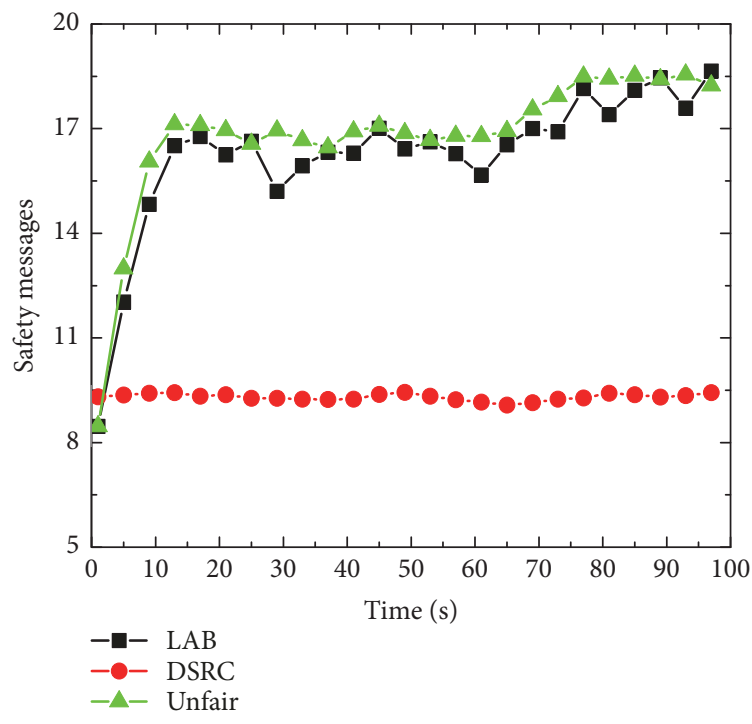

Figure 9: Time trend of safety messages.

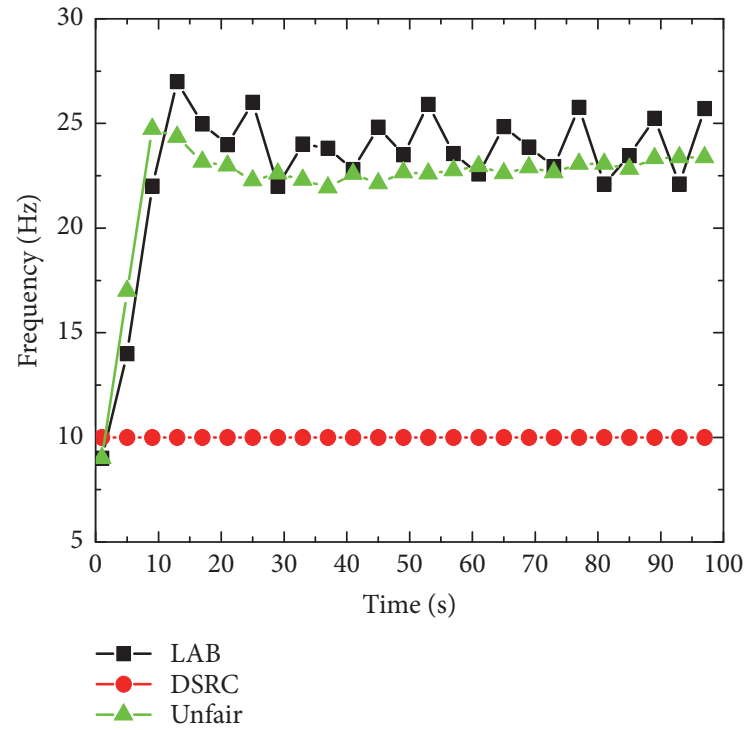

Figure 10: Time trend of broadcast frequency.

received safety messages of $\mathrm{LAB}$ increase to a stable level in 10 s.

Then we test the time trend of average broadcast frequency as shown in Figure 10. We can also find that average broadcast frequency of $\mathrm{LAB}$ converges to the optimal state in 10 s.

From previous results, we know that $\mathrm{LAB}$ is able to adapt to a changing environment and quickly converge to the optimal state.

5.4. Performance on Different Roads. In this subsection, we test performance of $\mathrm{LAB}$ on different roads. In this test, vehicle number ranges from 20 to 380 .

The results are shown in Figures 11 and 12. We can find that the general trend on these three roads is roughly the same.

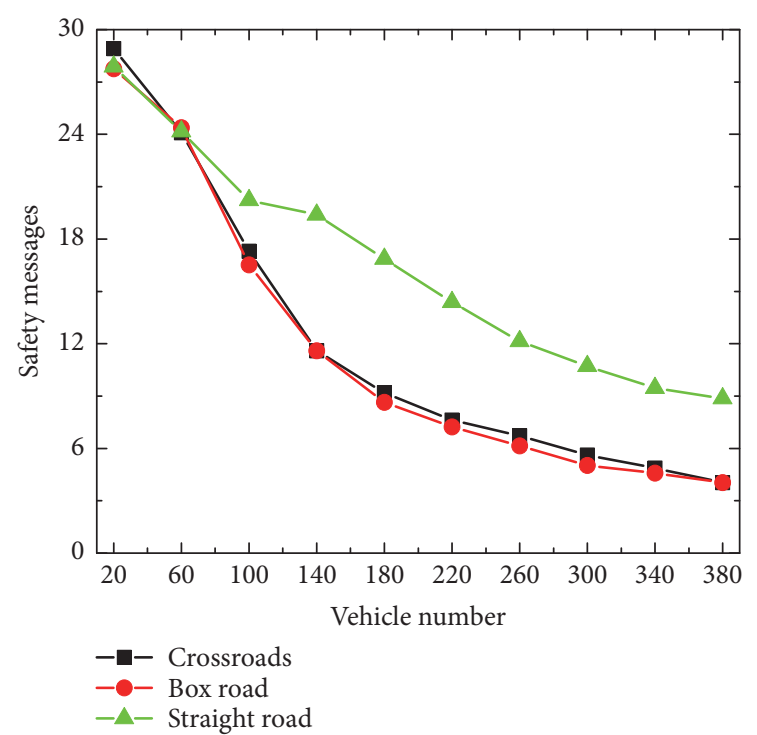

FIGURE 11: Safety messages on different roads.

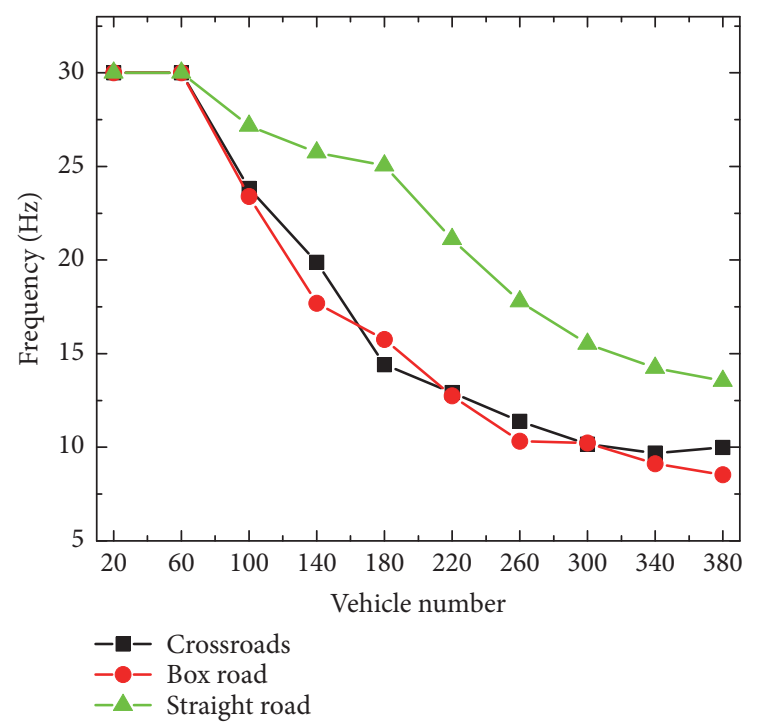

FIGURE 12: Frequency on different roads.

Furthermore, performance on straight road is better than box road and crossroads because the intersection of them is more likely to congest.

5.5. Performance on Different Packet Lengths. In this subsection, we check the performance changes of $\mathrm{LAB}$ on different packet lengths. In this test, 100 vehicles are moved in a crossroad being $1 \mathrm{~km}$ in each direction and the packet length changes from 50 Bytes to 700 Bytes.

The result is shown as in Figures 13 and 14. We find that the bigger the packet length, the fewer the safety messages that the vehicles can receive. It is because when packet length increases, it costs channel more time to send a packet. Therefore, broadcast frequency drops too. 


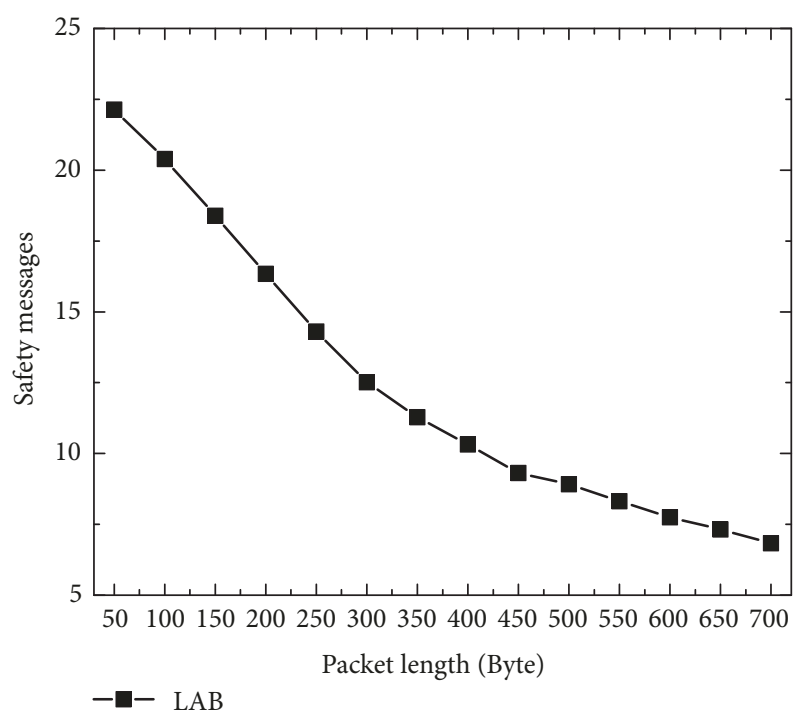

Figure 13: Safety messages on different packet lengths.

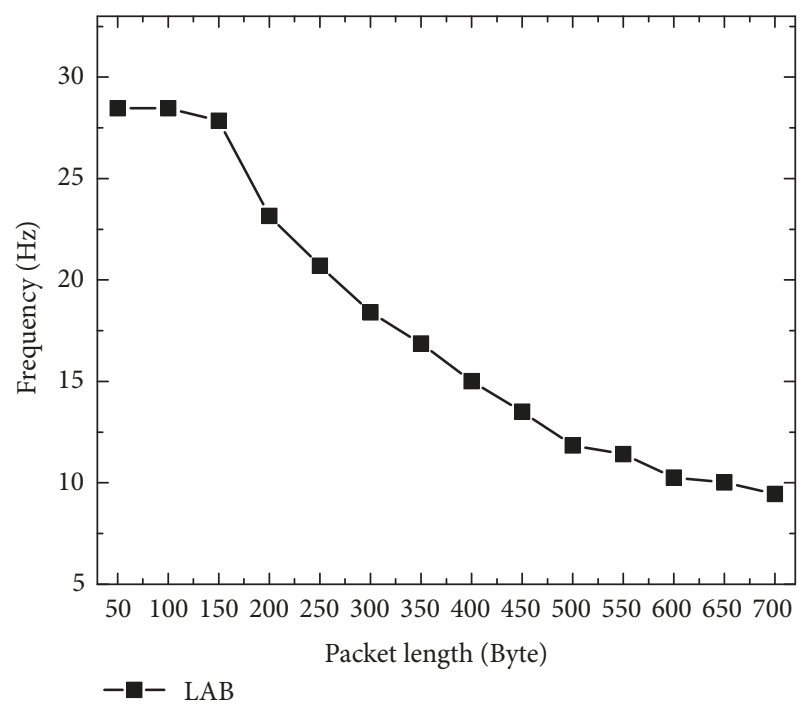

FIGURE 14: Frequency on different packet lengths.

5.6. Performance on Different Transmission Power. In this subsection, we check the performance changes of LAB on different transmission power. In this test, 100 vehicles are moved in a crossroad which is $1 \mathrm{~km}$ in each direction and the transmission power changes from $6 \mathrm{dBm}$ to $28 \mathrm{dBm}$.

We evaluate the frequency and average received packet number in Figures 15 and 16. We find that when the transmission power increases, broadcast frequency and the number of safety messages converge into a stable state.

5.7. Time Trend of Different Time Window. In this subsection, we check the time trend of LAB on different time window (twnd). In this test, 100 vehicles are moved in a crossroad which is $1 \mathrm{~km}$ in each direction.

In Figures 17 and 18, the safety messages and frequency are evaluated, respectively. Four curves shown in figures

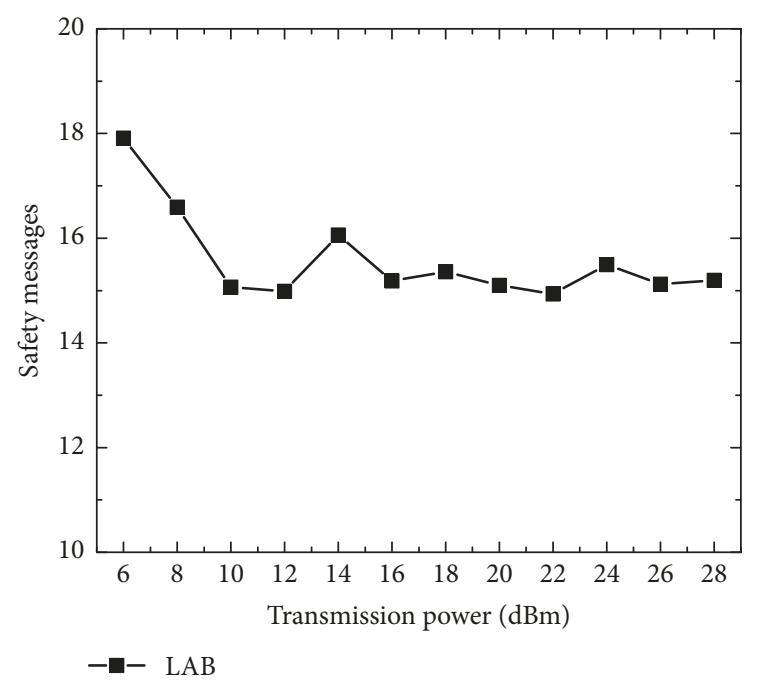

FIGURE 15: Safety messages on different transmission power.

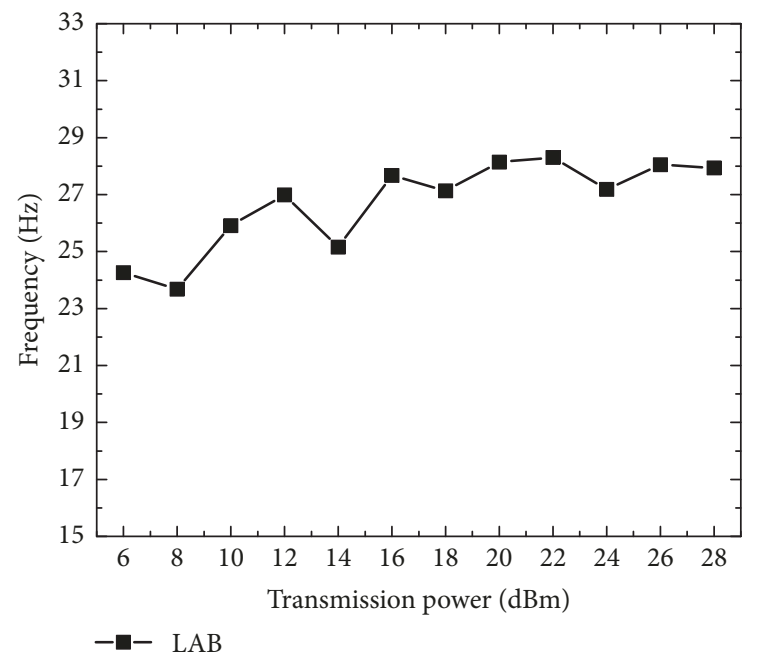

Figure 16: Frequency on different transmission power.

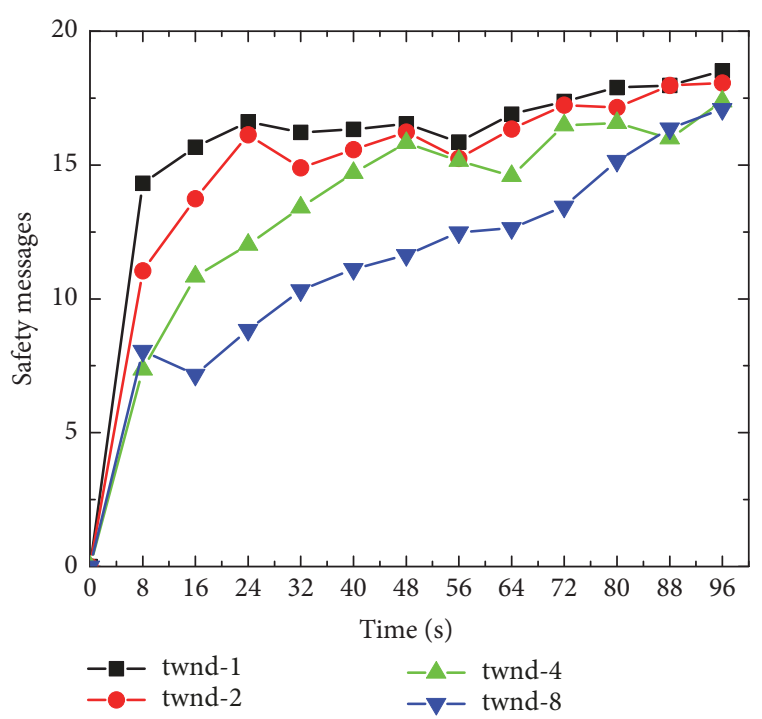

FIGURE 17: Time trend of safety messages. 


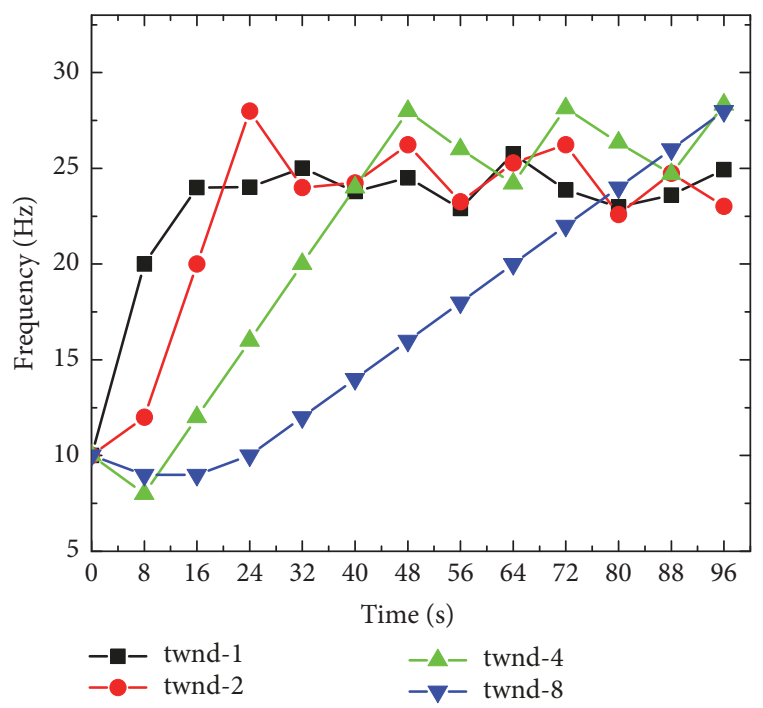

FIGURE 18: Time trend of broadcast frequency.

represent 4 sizes of twnd, which are $1 \mathrm{~s}, 2 \mathrm{~s}, 4 \mathrm{~s}$, and $8 \mathrm{~s}$. We find that the convergence speed drops when twnd becomes larger. Therefore, choosing $1 \mathrm{~s}$ as the default setting of twnd is proper, which is tradeoff between overhead and sensibility.

\section{Conclusion}

In this paper, we propose lightweight adaptive broadcast control (LAB) for DSRC safety message. LAB consists of broadcast manager, transmitter controller, and channel detector. To adjust broadcast frequency, broadcast manager will analyse channel conditions of neighboring vehicles and adjust frequency correspondingly. If channel is congested, broadcast frequency will be reduced till it gets to lower bound. If channel is idle, broadcast frequency will increase till it gets to upper bound. Furthermore, channel condition is assessed by channel detector and LAB will broadcast its channel condition along with safety message. We conduct extensive simulations to evaluate the performance of LAB and simulation results validate efficiency of our proposed scheme.

\section{Data Availability}

The data used to support the findings of this study are available from the corresponding author upon request.

\section{Conflicts of Interest}

The authors declare that there are no conflicts of interest regarding the publication of this article.

\section{Acknowledgments}

This work is partly supported by National Key Research and Development Program grant (2016YFE0100600), NSFC (61672349, 61672353čň61472252), and China 973 Project (2014CB340303).

\section{References}

[1] L. Atzori, A. Iera, and G. Morabito, "The internet of things: a survey," Computer Networks, vol. 54, no. 15, pp. 2787-2805, 2010.

[2] D. Serpanos and M. Wolf, "Industrial internet of things," in Internet-of-Things (IoT) Systems, pp. 37-54, Springer, 2018.

[3] X. Li, D. Li, J. Wan, A. V. Vasilakos, C.-F. Lai, and S. Wang, "A review of industrial wireless networks in the context of Industry 4.0," Wireless Networks, vol. 23, no. 1, pp. 23-41, 2017.

[4] Q. Liu, J. Wan, and K. Zhou, "Cloud manufacturing service system for industrial-cluster-oriented application," Journal of Internet Technology, vol. 15, no. 3, pp. 373-380, 2014.

[5] P. Mell and T. Grance, "The NIST definition of cloud computing," Cloud Computing and Government: Background, Benefits, Risks, pp. 171-173, 2011.

[6] C. E. Jones, K. M. Sivalingam, P. Agrawal, and J. C. Chen, "A survey of energy efficient network protocols for wireless networks," Wireless Networks, vol. 7, no. 4, pp. 343-358, 2001.

[7] S. J. Russell and P. Norvig, Artificial intelligence: a modern approach, Malaysia; Pearson Education Limited, 2016.

[8] L. Kong, M. K. Khan, F. Wu, G. Chen, and P. Zeng, "Millimeter-wave wireless communications for IoT-cloud supported autonomous vehicles: Overview, design, and challenges," IEEE Communications Magazine, vol. 55, no. 1, pp. 62-68, 2017.

[9] S. Zeadally, R. Hunt, Y.-S. Chen, A. Irwin, and A. Hassan, "Vehicular ad hoc networks (VANETS): status, results, and challenges," Telecommunication Systems, vol. 50, no. 4, pp. 217241, 2012.

[10] J. B. Kenney, "Dedicated short-range communications (DSRC) standards in the United States," Proceedings of the IEEE, vol. 99, no. 7, pp. 1162-1182, 2011.

[11] F. Bai, D. D. Stancil, and H. Krishnan, "Toward understanding characteristics of dedicated short range communications (DSRC) from a perspective of vehicular network engineers," in Proceedings of the 16th Annual Conference on Mobile Computing and Networking (MobiCom '10), pp. 329-340, ACM, September 2010.

[12] X. Chen, L. Kong, X. Liu, L. Rao, F. Bai, and Q. Xiang, "How cars talk louder, clearer and fairer: Optimizing the communication performance of connected vehicles via online synchronous control," in Proceedings of the 35th Annual IEEE International Conference on Computer Communications, IEEE INFOCOM 2016, USA, April 2016.

[13] G. Bansal, J. B. Kenney, and C. E. Rohrs, "LIMERIC: a linear adaptive message rate algorithm for DSRC congestion control," IEEE Transactions on Vehicular Technology, vol. 62, no. 9, pp. 4182-4197, 2013.

[14] S. Al-Sultan, M. M. Al-Doori, A. H. Al-Bayatti, and H. Zedan, "A comprehensive survey on vehicular Ad Hoc network," Journal of Network and Computer Applications, vol. 37, no. 1, pp. 380-392, 2014.

[15] F. Martelli, M. Elena Renda, G. Resta, and P. Santi, "A measurement-based study of beaconing performance in IEEE 802.11p vehicular networks," in Proceedings of the IEEE Conference on Computer Communications, INFOCOM 2012, pp. 15031511, USA, March 2012.

[16] Z. Tong, H. Lu, M. Haenggi, and C. Poellabauer, "A Stochastic Geometry Approach to the Modeling of DSRC for Vehicular Safety Communication," IEEE Transactions on Intelligent Transportation Systems, vol. 17, no. 5, pp. 1448-1458, 2016.

[17] T. V. Nguyen, F. Baccelli, K. Zhu, S. Subramanian, and X. Wu, "A performance analysis of CSMA based broadcast protocol in 
VANETs," in Proceedings of the IEEE INFOCOM, pp. 2805-2813, Turin, Italy, April 2013.

[18] F. Ye, R. Yim, J. Zhang, and S. Roy, "Congestion control to achieve optimal broadcast efficiency in VANETs," in Proceedings of the 2010 IEEE International Conference on Communications, ICC 2010, South Africa, May 2010.

[19] S. Djahel and Y. Ghamri-Doudane, "A robust congestion control scheme for fast and reliable dissemination of safety messages in VANETs," in Proceedings of the IEEE Wireless Communications and Networking Conference (WCNC '12), pp. 2264-2269, Shanghai, China, April 2012.

[20] H. Lv, X. Ye, L. An, and Y. Wang, "Distributed beacon frequency control algorithm for VANETs (DBFC)," in Proceedings of the 2nd International Conference on Intelligent Systems Design and Engineering Applications (ISDEA '12), pp. 243-246, January 2012.

[21] M. S. Bouassida and M. Shawky, "On the congestion control within VANET," in Proceedings of the 2008 1st IFIP Wireless Days, WD 2008, UAE, November 2008.

[22] M. A. Benatia, L. Khoukhi, M. Esseghir, and L. M. Boulahia, "A markov chain based model for congestion control in VANETs," in Proceedings of the 27th International Conference on Advanced Information Networking and Applications Workshops, WAINA 2013, pp. 1021-1026, Spain, March 2013.

[23] B. M. Mughal, A. A. Wagan, and H. Hasbullah, "Efficient congestion control in VANET for safety messaging," in Proceedings of the 2010 International Symposium on Information Technology, ITSim'10, pp. 654-659, Malaysia, June 2010.

[24] G. Bansal and J. B. Kenney, "Achieving weighted-fairnessin message rate-based congestion control for DSRC systems," in Proceedings of the IEEE 5th International Symposium on Wireless Vehicular Communications (WiVeC '13), pp. 1-5, Dresden, Germany, June 2013.

[25] T. Tielert, D. Jiang, Q. Chen, L. Delgrossi, and H. Hartenstein, "Design methodology and evaluation of rate adaptation based congestion control for vehicle safety communications," in Proceedings of the IEEE Vehicular Networking Conference (VNC '11), pp. 116-123, November 2011.

[26] G. Bansal, H. Lu, J. B. Kenney, and C. Poellabauer, "EMBARC: Error model based adaptive rate control for vehicle-to-vehicle communications," in Proceedings of the 10th ACM International Workshop on Vehicular Inter-Networking, Systems, and Applications, VANET 2013, pp. 41-50, Taiwan, June 2013.

[27] “ns-3," https://www.nsnam.org/.

[28] "SUMO - Simulation of Urban MObility," http://sumo.dlr.de.

[29] Y. P. Fallah, H. ChingLing, S. Raja, and H. Krishnan, "Congestion control based on channel occupancy in vehicular broadcast networks," in Proceedings of the IEEE 72nd Vehicular Technology Conference Fall (VTC-Fall '10), pp. 1-5, Ottawa, Canada, September 2010.

[30] J. Yin, T. Elbatt, G. Yeung et al., "Performance evaluation of safety applications over DSRC vehicular ad hoc networks," in Proceedings of the 1st ACM International Workshop on Vehicular Ad Hoc Networks (VANET '04), pp. 1-9, October 2004. 


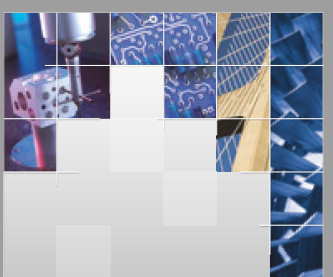

\section{Enfincering}
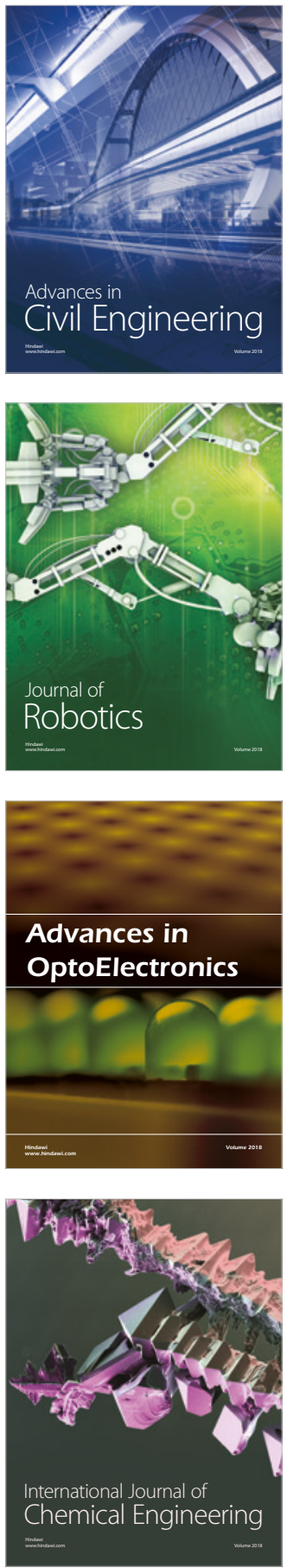

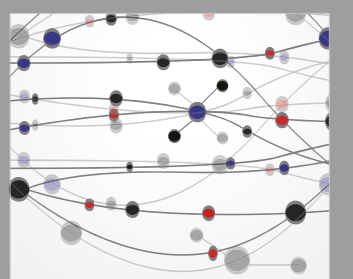

\section{Rotating \\ Machinery}

The Scientific World Journal

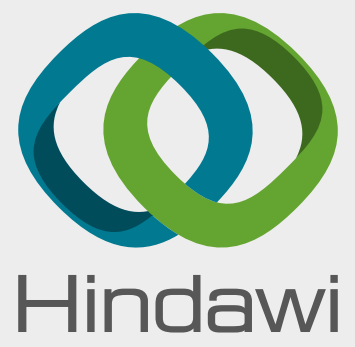

Submit your manuscripts at

www.hindawi.com
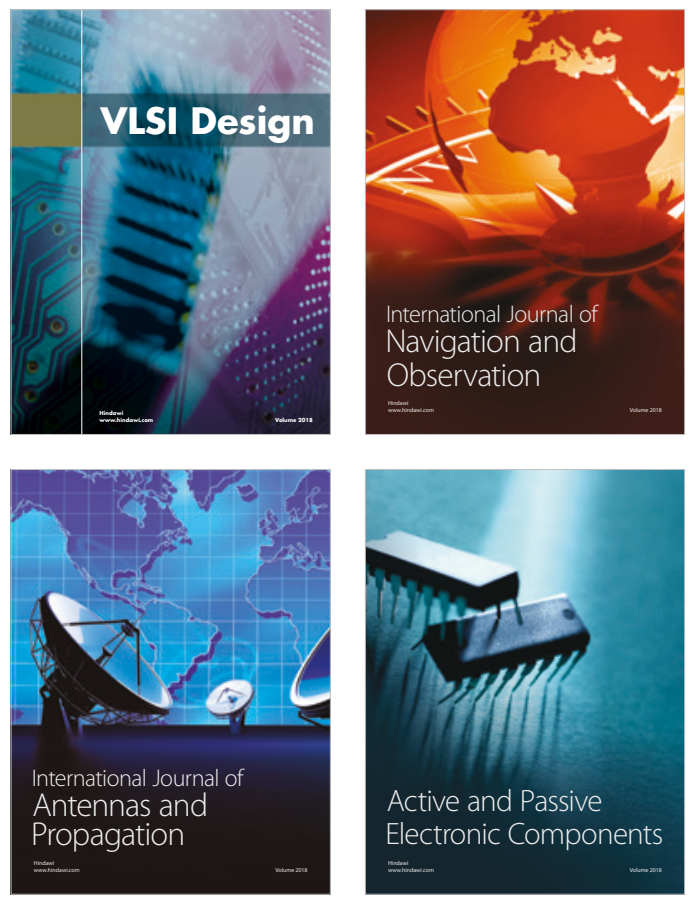
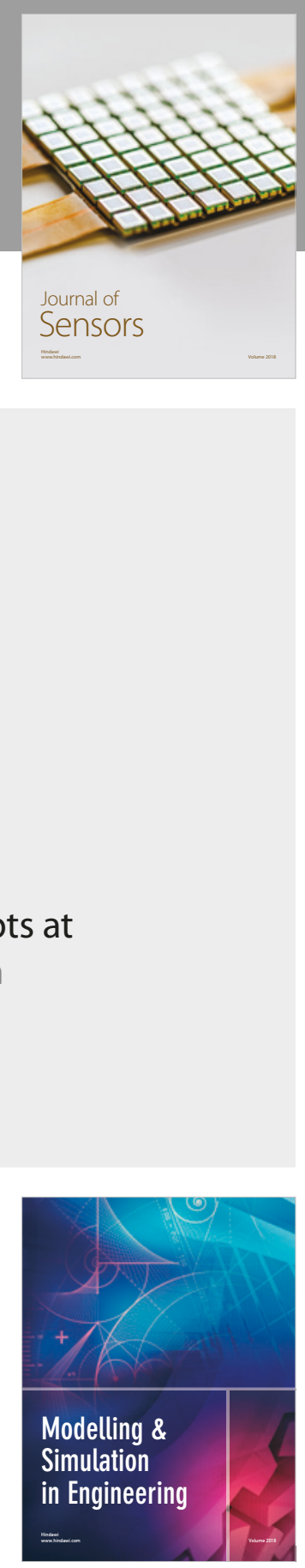

\section{Advances \\ Multimedia}
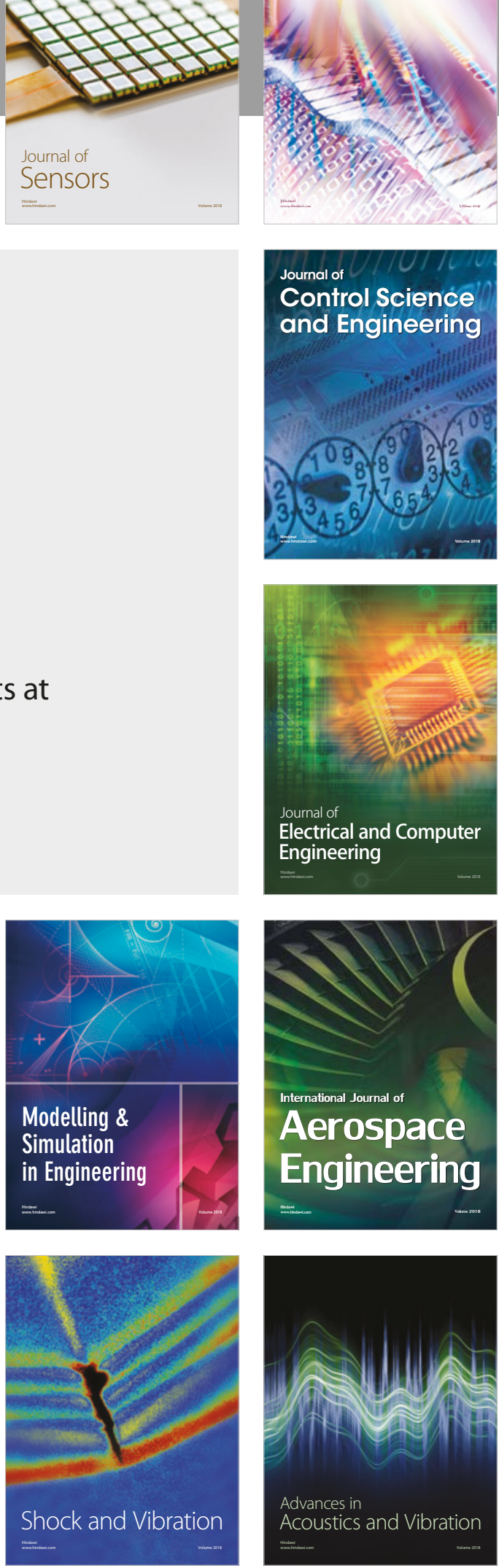